\section{Re: Maternal Depressive Symptomatology: 16-Month Follow-up of Infant and Maternal Health-Related Quality of Life}

To the Editor: As a Doctor of Nursing Practice working as Nursing Unit Director in a maternal/newborn setting at an academic medical center, I read with great interest Darcy et al's ${ }^{1}$ recent article "Maternal Depressive Symptomatology: 16-Month Follow-up of Infant and Maternal Health-Related Quality of Life." The authors should be commended for researching the long-term effects of postpartum mood disorders after new mothers' transition home from the hospital setting. I am impressed this study is a part of the Weaving Work and Family Project: Implications for Mother and Child. ${ }^{2}$ For nurse clinicians and managers, postpartum mood disorders are a challenging aspect of maternal newborn care. Hillerer et $\mathrm{al}^{3}$ propose that maternal adaptations, such as decreased anxiety and attenuated stress responsiveness, are necessary to enable successful postnatal development of the offspring.

Many devastating consequences of postpartum mood disorder could be avoided with early identification and referral to psychosocial support in the pre- and postnatal settings. There are multiple opportunities for health care providers to monitor and intervene in the prenatal setting when there is evidence of maternal stressors. For example, in the article Darcy et al identify significant depressive symptomatology among African American women aged 18 to 24 years who are impoverished and unwed. Tarantino et $\mathrm{al}^{4}$ establish that the etiology of complex psychiatric disorders result from both genetics and the environment. No definitive environmental factor has been implicated, but studies suggest that deficits in maternal bonding many be an important contributing factor in the development of anxiety and depression. It is possible that neither race nor socioeconomic statuses are factors in assessing or predicting postpartum mood disorders.

Another aspect discussed in the article is an increase in newborn diarrhea and frequent visits to the pediatrician. Talge et $\mathrm{al}^{5}$ determined that maternal and antenatal stress is associated with adverse neurobehavioral outcomes in off spring. including both social/emotional and cognitive functioning during childhood and later in life. I recommend that we inform prenatal patients about work and life stressors and how to address them. Some of the most tragic stories in the media pertain to postpartum mood disorders undetected in the pre- and postnatal settings and the outcome of infanticide. Further research is needed to determine if observed research is causal. Such research is beneficial if more interdisciplinary teams study this problem. Regardless of the mother's ethnicity, the prenatal setting is key to the prevention of negative outcomes of postpartum mood disorders in the community, and it is an opportune setting in which to provide counseling and education to assist childbearing women in making informed choices about postpartum mood disorders once the diagnosis is known. Using best practices to support childbearing families is a high priority. Darcy et $\mathrm{al}^{1}$ are commended for the longitudinal study on postpartum mood disorders and the quality of life for both mother and baby.

\section{Debra Griffin Stevens, DNP, RN Covington, GA debragstevens@bellsouth.net}

\section{References}

1. Darcy JM, Grzywacz JG, Stephens RL, Leng I, Clinch CR, Arcury TA. Maternal depressive symptomatology: 16 month follow-up of infant and maternal health-related quality of life. J Am Board Fam Med 2011;24(3):249-57.

2. Gryzwacz J. Weaving Work and Family Project: implications for mother and child. 2010.

3. Hillerer K, Reber S, Neuman I, Slatter D. Exposure to chronic pregnancy stress reverses peripartum-associated adaptations: implications for postpartum anxiety and mood disorders. Endocrinology 2011;152:3930-40.

4. Tarantino L, Sullivan P, Meltzer-Brody S. Using animal models to disentangle the role of genetic, epigenetic, and environmental influences on behavioral outcomes associated with maternal anxiety and depression. Front Psychiatry 2011;2:44.

5. Talge N, Neal C, Glover V. Early stress, translational research and prevention science network: fetal and neonatal experience on child and adolescent mental health. Journal of Child Psychology 2007.

doi: 10.3122/jabfm.2012.03.120008

The authors of the original manuscript declined to respond to this letter.

\section{Re: Irritable Bowel Syndrome: A "Mesh" of a Situation}

To the Editor: We read with great interest Dr. Barnes' ${ }^{1}$ brief report and literature review in the January/February 2012 issue of the Fournal of the American Board of Family Medicine. He describes a fascinating case in which the symptoms of irritable bowel syndrome (IBS) result from a rare mechanical cause: mesh herniorrhaphy. He demonstrates the value of meticulous history taking, examination, and investigation in patients with IBS, particularly if medical management proves ineffective. Dr. Barnes' case acts as a reminder of the challenges that diagnoses of IBS (and other diagnoses of exclusion) pose, and we believe it is pertinent to briefly elaborate on this. 